\title{
Competencias científicas en la enseñanza y el aprendizaje por investigación. Un estudio de caso sobre corrosión de metales en secundaria
}

\section{Scientific competences in teaching and learning through research.} A case study about the corrosion of metals in secondary education

\author{
Antonio Joaquín Franco-Mariscal \\ IES Juan Ramón Jiménez, Málaga, España. \\ Universidad de Málaga, Málaga, España. \\ antoniojoaquin.franco@uma.es
}

RESUMEN • Este artículo plantea un enfoque alternativo para el desarrollo de la competencia científica en una enseñanza-aprendizaje por investigación en educación secundaria, a partir de una situación contextualizada. Según el enfoque propuesto, la competencia científica tiene siete dimensiones: planteamiento de la investigación; manejo de la información; planificación y diseño de la investigación; recogida y procesamiento de datos; análisis de datos y emisión de conclusiones; comunicación de resultados, y actitud o reflexión crítica y trabajo en equipo. Esta propuesta resalta algunos aspectos que a menudo no son suficientemente tenidos en cuenta en la enseńanza de las Ciencias como el manejo de la información, la comunicación de resultados y la actitud o reflexión crítica y el trabajo en equipo. El enfoque se ejemplifica con un estudio de caso con estudiantes de educación secundaria obligatoria. Finalmente, se hacen algunas consideraciones sobre la importancia del enfoque y la necesidad de una formación del profesorado en trabajos de investigación escolares.

PALABRAS CLAVE: enseñanza-aprendizaje por investigación; competencia científica; trabajos de investigación; estudio de caso; corrosión.

ABSTRACT - An alternative approach to the development of the scientific competence in a teaching and learning by research in secondary education from a contextualized situation is presented in this paper. According to the approach, the scientific competence has seven dimensions: proposal of research; information management; planning and research design; collection and processing of data; data analysis and drawing conclusions; communication of results; and critical attitude/thinking and ability to work as a part of a team. Some aspects as information management, communication of results, and critical attitude/thinking and ability to work as a part of a team, that are often not sufficiently considered in the teaching of science are emphasised in this proposal. The approach is illustrated with examples in a case study with compulsory secondary students. Finally, some considerations about the importance of the approach and the need of a teacher training in school research works are made.

KEYWORDS: teaching and learning by research; scientific competence; research works; case study; corrosion.

Fecha de recepción: diciembre 2014 • Aceptado: abril 2015

Franco-Mariscal, A. J. (2015) Competencias científicas en la enseñanza y el aprendizaje por investigación. Un estudio de caso sobre corrosión de metales en secundaria. Enseñanza de las Ciencias, 33.2, pp. 231-252 


\section{COMPETENCIAS CIENTÍFICAS Y TRABAJOS DE INVESTIGACIÓN}

Desde hace más de una década, las propuestas de la OCDE (2002) y posteriormente de la Unión Europea (UE, 2006) aconsejaron un nuevo enfoque de enseñanza-aprendizaje basado en competencias que rápidamente se extendió a los diferentes sistemas educativos europeos, entre ellos el de España. Este enfoque presta especial atención al desarrollo de competencias clave en los estudiantes de secundaria (OCDE, 2009), entendiéndose estas como una combinación de conocimientos, habilidades y actitudes apropiadas para desenvolverse adecuadamente en distintos contextos de la vida diaria (UE, 2006). Entre estas competencias clave y dentro del currículo español (MEC, 2007) se encuentra la denominada competencia en el conocimiento y la interacción con el mundo físico y natural, en adelante competencia científica.

El enfoque de las competencias constituye, sin duda, una nueva oportunidad para dar respuestas colectivas a los problemas de enseñanza-aprendizaje de las Ciencias (Pro, 2011) y para la mejora de las clases de ciencias (Jiménez-Aleixandre, 2009). Asimismo presenta una nueva ocasión para extender unas prácticas hasta ahora minoritarias, y para hacer una reflexión sobre la manera en la que estamos ejerciendo la profesión docente (Sanmartí, 2010). En definitiva, se trata de una situación ideal para orientar la enseñanza de las Ciencias hacia propuestas que emanan de las innovaciones e investigaciones educativas y contribuir, de esta forma, a su consolidación (Vilches y Gil, 2010).

Desde la enseñanza de las Ciencias se han planteado diferentes enfoques para caracterizar la competencia científica, entre las que destacan la propuesta de PISA en el marco de la evaluación (OCDE, 2006) (identificación de cuestiones científicas, explicación científica de fenómenos y utilización de pruebas científicas), la aportación del Ministerio de Educación (MEC, 2006, 2007) o de las distintas comunidades autónomas en los currículos de primaria y secundaria, o el enfoque reciente de Pedrinaci y Cañal (Pedrinaci et al., 2012; Cañal, 2012) que considera cuatro dimensiones de la competencia científica (conceptual, metodológica, actitudinal e integrada).

En todas estas aportaciones, de alguna manera se cree conveniente que los estudiantes desarrollen algunas capacidades relacionadas con la investigación en el contexto escolar, como propone de una manera más directa la dimensión metodológica del enfoque de Pedrinaci y Cañal (Pedrinaci et al., 2012; Cañal, 2012).

En este sentido, el objetivo de la enseñanza-aprendizaje por investigación es acercar al estudiante al contexto en el que trabajan los científicos. En este punto debemos distinguir entre la investigación que se realiza en la "ciencia de los científicos» y en el ámbito escolar. Mientras en el primer caso los científicos abordan problemas que no se han resuelto, en el contexto escolar las investigaciones que se plantean a los alumnos son problemas dirigidos de los que los docentes conocen sus posibles soluciones y el marco teórico en el que se sustentan. A pesar de ello, la investigación en el medio escolar, centrada en el alumno con un papel activo en el aprendizaje, es compatible y adecuada dentro de un marco constructivista. Debemos indicar que la conveniencia de orientar el aprendizaje de las Ciencias a través de la investigación en la escuela y de situaciones problemáticas ha sido un tópico resaltado por diferentes autores (Gil, 1986; 1993; Burbules y Linn, 1991; Wheatley, 1991; Belmonte, 2011; Caamaño, 2012; Franco-Mariscal, 2015), incluso desde mucho antes de la aparición del marco de las competencias.

Sin duda, la indagación, entendida como las estrategias de enseñanza-aprendizaje que permiten aprender Ciencia a partir de la realización de investigaciones que aporten evidencias experimentales con la finalidad de promover la génesis y la evolución del conocimiento científico escolar, se considera hoy día una de las líneas de investigación más fructíferas y debatidas en Didáctica de las ciencias.

Según Ferrés, Marbà y Sanmartí (2015), la utilización de la indagación en las actividades escolares puede constituir un elemento de innovación y progreso hacia modelos de Didáctica de las ciencias no centrados exclusivamente en la transmisión de conocimientos, y que persiguen los objetivos del enfoque competencial de la enseñanza. Asimismo, este tipo de estrategia potencia el desarrollo de 
competencias científicas y ofrece al estudiante un horizonte mucho más significativo para su futura vida profesional (García y Ladino, 2008). Tales son las posibilidades educativas de la indagación que el documento Science Education now (Rocard, 2007) ha propuesto su uso como modelo didáctico para combatir la desmotivación en Ciencias.

A pesar de la potencialidad de la indagación (Caamaño, 2012), la realidad docente muestra cómo la metodología que aplicamos la mayoría de los profesores está bastante alejada de una enseñanza de las Ciencias basada en este enfoque, probablemente porque, como se ha indicado, una parte fundamental del currículo se centra en transmitir ideas de la ciencia, sin incorporar ideas sobre la ciencia, relativas a cómo se ha obtenido ese conocimiento (Fernández-López, 2011). Por ello, la realización de trabajos de investigación en el contexto escolar es poco habitual en la etapa obligatoria de la educación secundaria (12-16 años), aunque sí algo más frecuente entre los alumnos de bachillerato (17-18 años), especialmente a partir de la inclusión de este tipo de trabajos en el currículo de algunas comunidades autónomas como Cataluña o Murcia.

A todo esto debemos sumar que son pocas las ocasiones en las que los profesores proponemos a los alumnos actividades de indagación verdaderas (Tamir y García, 1992), es decir, trabajos de investigación con un nivel de dificultad alto en los que la indagación esté totalmente abierta, y en los que sea el propio estudiante el que dirige todo el proceso, desde el planteamiento de interrogantes y la elección de los métodos para dar respuesta al problema, hasta la recolección de datos y su posterior análisis (Bell, Maeng y Peters, 2010).

En este sentido, el Programa Andalucía Profundiza (Junta de Andalucía, 2010) trabaja en esta línea de promoción de la realización de proyectos de investigación con alumnado de secundaria obligatoria en horario extraescolar. Entre sus objetivos se encuentran la profundización en el conocimiento del alumnado; estimular su interés por la investigación en cualquier ámbito mediante la adquisición de estrategias que le permitan la adquisición y mejoras de sus competencias a lo largo de toda la vida; incentivar la autonomía e iniciativa personal del estudiante estimulando la innovación, la creatividad y la originalidad, y contribuir al cambio en las prácticas docentes favoreciendo la promoción de nuevas metodologías que fomenten el desarrollo de las competencias clave del alumnado. En resumen, este programa está permitiendo que en la actualidad muchos estudiantes andaluces estén adquiriendo competencias a través de trabajos de investigación en la escuela en el máximo nivel de indagación que comentamos.

En definitiva, el desarrollo de competencias científicas a través de investigaciones a nivel escolar debe considerarse como un factor clave para tener en cuenta, por lo que creemos conveniente definir claramente cuáles deberían ser las competencias científicas que un estudiante puede desarrollar en una enseñanza-aprendizaje por investigación. Por ello, el objetivo de este artículo es presentar una propuesta de competencias científicas que el alumnado de secundaria puede adquirir en un trabajo de investigación, así como ejemplificarlas a través de un caso concreto. Se insiste de nuevo en utilizar enfoques concretos de la competencia científica tanto en el diseño de tareas de aprendizaje (FrancoMariscal, Blanco-López y España-Ramos, 2014) como en actividades experimentales y trabajos de investigación escolares.

\section{REVISIÓN DE LA LITERATURA SOBRE COMPETENCIAS CIENTÍFICAS EN INVESTIGACIONES ESCOLARES}

Está claro que cuando el alumno debe desenvolverse en una investigación escolar tiene que demostrar que posee, o que desarrollará, un conjunto de habilidades o destrezas interrelacionadas entre sí y que se pondrán de manifiesto como distintas competencias producto de la práctica, la teoría, la experiencia y el contexto (Vargas, 2002). 
Pero, ¿de qué competencias científicas estamos hablando al realizar trabajos de investigación en el contexto escolar? Cabe pensar que las competencias científicas en un enfoque de enseñanza-aprendizaje por investigación deben tener una estrecha relación con los objetivos que orientan el desarrollo de una investigación científica. Desde nuestro punto de vista, las competencias científicas de la investigación escolar deben corresponderse con las diferentes capacidades que el estudiante va adquiriendo al ir avanzando en su investigación, y que va plasmando al desarrollar cada una de sus fases, de forma similar a como lo hacen los científicos al justificar el problema, formular objetivos e hipótesis, elaborar un marco teórico y revisar los antecedentes, elaborar un diseño metodológico, presentar y tratar los datos, discutir o valorar los resultados, o emitir conclusiones, pero no olvidemos que siempre bajo las orientaciones de su profesor.

Para intentar responder a la pregunta anteriormente expuesta, haremos un análisis de algunas propuestas interesantes de la literatura que o bien aluden directamente a algunas competencias científicas asociadas a la investigación escolar, o bien subyacen de ellas de manera implícita.

Entre los trabajos que aluden claramente al enfoque de las competencias destacan las propuestas de García y Ladino (2008) y Pedrinaci y Cañal (Pedrinaci et al., 2012; Cañal, 2012). García y Ladino (2008) distinguen dos tipos de competencias científicas en una investigación escolar: básicas e investigativas. Según estos autores, las competencias científicas básicas incluyen desempeños relacionados con procesos iniciales de reconocimiento de un lenguaje científico, desarrollo de habilidades experimentales, organización de la información y trabajo en grupo. La competencia científica investigativa incluye procesos cognitivos y sociales más allá de la selección y el procesamiento de la información o del saber disciplinar, y tiene un carácter más procedimental al permitir que el alumno integre de manera creativa y propositiva los conocimientos en su interacción crítica frente a nuevas situaciones y que resuelva problemas construyendo significados contextualizados. Proponen asimismo diferentes órdenes de complejidad para poder evaluar adecuadamente cada una de estas competencias científicas.

La dimensión metodológica de la competencia científica de Pedrinaci y Cañal (Pedrinaci et al., 2012; Cañal, 2012) destaca la capacidad del alumno para identificar problemas científicos y diseñar estrategias para su investigación, la capacidad para obtener información relevante para la investigación, la capacidad para procesar la información obtenida y la capacidad para formular conclusiones fundamentadas. Asimismo propone, dentro de la dimensión actitudinal, la capacidad para valorar la calidad de una información en función de su procedencia y de los procedimientos utilizados para generarla, también muy presente al realizar una investigación.

Por su parte, Gil (1993) propuso un diagrama de investigación a partir de situaciones problemáticas abiertas en las que el alumno puede participar en la construcción del conocimiento. En este diagrama se hace referencia a la necesidad de introducir aspectos de la investigación científica como el enunciado preciso del problema, la construcción y fundamentación de modelos e hipótesis contrastables, la necesidad de elaborar estrategias diversas de contrastación, o la interpretación y comunicación de los resultados.

En la literatura también podemos encontrar diferentes instrumentos de evaluación (Tamir, Nussinovitz y Friedler, 1982; Ferrés et al., 2015) para valorar la capacidad de los alumnos para comprender y aplicar investigaciones. Desde nuestro punto de vista, las categorías propuestas en estos instrumentos pueden interpretarse en términos de competencias científicas que el estudiante debería ser capaz de desarrollar en una investigación escolar. Así, el Practical Test Assessment Inventory (PTAI) de Tamir et al. (1982), diseñado para evaluar actividades prácticas, consta de 21 categorías (Tabla 1) que abordan los aspectos de comprensión de los procesos de una investigación, como la identificación de problemas investigables, la capacidad de formular hipótesis, identificar las variables de un diseño experimental, analizar datos y extraer conclusiones. Asimismo incluye otras categorías relacionadas con aspectos más procedimentales de una investigación. Todas ellas pueden considerarse competencias científicas dentro 
de un trabajo de investigación en la escuela. Recientemente, a partir de este test y mediante la unificación de categorías, Ferrés et al. (2015) diseñaron un instrumento denominado «nuevo PTAI» (NPTAI) para evaluar trabajos de indagación en bachillerato, reduciendo los 21 ítems a 7 grandes categorías que recogen los principales aspectos de una investigación (tabla 1). Esta propuesta incluye como aspecto novedoso una categoría de metarreflexión que tiene como utilidad poder evaluar la comprensión del alumnado con respecto a los métodos de la Ciencia.

La tabla 1 establece una correspondencia entre las competencias científicas de estos cinco trabajos (García y Ladino, 2008; Pedrinaci et al., 2012; Gil, 1993; Tamir et al., 1982; Ferrés et al., 2015). Como se observa, las competencias recogidas en las propuestas analizadas son complementarias y constituyen una visión amplia de las competencias científicas que el alumnado puede desarrollar en una investigación.

Tabla 1.

Distintas propuestas de competencias científicas en una investigación escolar

\begin{tabular}{|c|c|c|c|c|c|}
\hline $\begin{array}{l}\text { Categorias del } \\
\text { instrumento de } \\
\text { evaluación NPTAI } \\
\text { (Ferrés et al.,2015) }\end{array}$ & $\begin{array}{l}\text { Categorias del } \\
\text { instrumento de } \\
\text { evaluación PTAI } \\
\text { (Tamir et al., } \\
\text { 1982) }\end{array}$ & $\begin{array}{l}\text { Diagrama de } \\
\text { investigación } \\
\text { (Gil, 1993) }\end{array}$ & $\begin{array}{l}\text { Competencias de la } \\
\text { dimensión metodo- } \\
\text { lógica } \\
\text { (Pedrinaci et al., } \\
\text { 2012; Cañal, } \\
\text { 2012) }\end{array}$ & $\begin{array}{l}\text { Competencias bá- } \\
\text { sicas (CB) e investi- } \\
\text { gativas (CI) } \\
\text { (García y Ladino, } \\
\text { 2008) }\end{array}$ & $\begin{array}{l}\text { Dimensiones de } \\
\text { la competencia } \\
\text { cientifica en la } \\
\text { investigación } \\
\text { (Franco-Mariscal) }\end{array}$ \\
\hline $\begin{array}{l}\text { Identificación } \\
\text { de problemas o } \\
\text { formulación de } \\
\text { preguntas }\end{array}$ & $\begin{array}{l}\text { Formulación de } \\
\text { problemas }\end{array}$ & $\begin{array}{l}\text { Enunciado preciso } \\
\text { del problema }\end{array}$ & $\begin{array}{l}\text { Identificar proble- } \\
\text { mas científicos }\end{array}$ & & \multirow[b]{2}{*}{$\begin{array}{l}\text { Planteamiento de } \\
\text { la investigación }\end{array}$} \\
\hline \multirow[t]{2}{*}{$\begin{array}{l}\text { Formulación de } \\
\text { hipótesis }\end{array}$} & $\begin{array}{l}\text { Formulación de } \\
\text { hipótesis }\end{array}$ & $\begin{array}{l}\text { Construcción y } \\
\text { fundamentación } \\
\text { de modelos o } \\
\text { hipótesis contras- } \\
\text { tables }\end{array}$ & & & \\
\hline & & $\begin{array}{l}\text { Análisis cualitativo } \\
\text { de trabajos biblio- } \\
\text { gráficos, toma de } \\
\text { decisiones }\end{array}$ & $\begin{array}{l}\text { Valorar la calidad } \\
\text { de una informa- } \\
\text { ción en función } \\
\text { de su procedencia } \\
\text { y de los procedi- } \\
\text { mientos utilizados } \\
\text { para generarla } \\
\text { (Dimensión acti- } \\
\text { tudinal) }\end{array}$ & & $\begin{array}{l}\text { Manejo de la } \\
\text { información }\end{array}$ \\
\hline
\end{tabular}




\begin{tabular}{|c|c|c|c|c|c|}
\hline $\begin{array}{l}\text { Categorias del } \\
\text { instrumento de } \\
\text { evaluación NPTAI } \\
\text { (Ferrés et al.,2015) }\end{array}$ & $\begin{array}{l}\text { Categorias del } \\
\text { instrumento de } \\
\text { evaluación PTAI } \\
\text { (Tamir et al., } \\
\text { 1982) }\end{array}$ & $\begin{array}{l}\text { Diagrama de } \\
\text { investigación } \\
\text { (Gil, 1993) }\end{array}$ & $\begin{array}{l}\text { Competencias de la } \\
\text { dimensión metodo- } \\
\text { lógica } \\
\text { (Pedrinaci et al., } \\
\text { 2012; Cañal, } \\
\text { 2012) }\end{array}$ & $\begin{array}{l}\text { Competencias bá- } \\
\text { sicas }(C B) \text { e investi- } \\
\text { gativas (CI) } \\
\text { (Garcia y Ladino, } \\
\text { 2008) }\end{array}$ & $\begin{array}{l}\text { Dimensiones de } \\
\text { la competencia } \\
\text { cientifica en la } \\
\text { investigación } \\
\text { (Franco-Mariscal) }\end{array}$ \\
\hline \multirow{2}{*}{$\begin{array}{l}\text { Identificación de } \\
\text { variables }\end{array}$} & $\begin{array}{l}\text { Identificación de } \\
\text { la variable depen- } \\
\text { diente }\end{array}$ & & & & \multirow{6}{*}{$\begin{array}{l}\text { Planificación y } \\
\text { diseño de la inves } \\
\text { tigación }\end{array}$} \\
\hline & $\begin{array}{l}\text { Identificación de } \\
\text { la variable inde- } \\
\text { pendiente }\end{array}$ & & & & \\
\hline \multirow{4}{*}{$\begin{array}{l}\text { Planificación de la } \\
\text { investigación }\end{array}$} & $\begin{array}{l}\text { Planificación del } \\
\text { grupo de control }\end{array}$ & & & & \\
\hline & $\begin{array}{l}\text { Adecuación de la } \\
\text { experiencia al pro- } \\
\text { blema formulado }\end{array}$ & & & & \\
\hline & $\begin{array}{l}\text { Planificación } \\
\text { completa de la } \\
\text { experimentación }\end{array}$ & $\begin{array}{l}\text { Elaboración de } \\
\text { estrategias diversas } \\
\text { de contrastación, } \\
\text { incluyendo en } \\
\text { su caso el diseńo } \\
\text { y realización de } \\
\text { experimentos }\end{array}$ & $\begin{array}{l}\text { Diseñar estrategias } \\
\text { para la investiga- } \\
\text { ción }\end{array}$ & & \\
\hline & $\begin{array}{l}\text { Comprensión } \\
\text { de la función } \\
\text { del control en la } \\
\text { experiencia }\end{array}$ & & & & \\
\hline \multirow{9}{*}{$\begin{array}{l}\text { Recogida y proce- } \\
\text { samiento de datos }\end{array}$} & $\begin{array}{l}\text { Informe de los } \\
\text { resultados }\end{array}$ & $\begin{array}{l}\text { Realización de } \\
\text { experimentos }\end{array}$ & $\begin{array}{l}\text { Obtener informa- } \\
\text { ción relevante para } \\
\text { la investigación }\end{array}$ & $\begin{array}{l}\text { Elaborar y pre- } \\
\text { sentar informes } \\
\text { escritos (CB) }\end{array}$ & \multirow{9}{*}{$\begin{array}{l}\text { Recogida y } \\
\text { procesamiento de } \\
\text { datos }\end{array}$} \\
\hline & $\begin{array}{l}\text { Construcción de } \\
\text { gráficas }\end{array}$ & & \multirow[t]{2}{*}{$\begin{array}{l}\text { Procesar la infor- } \\
\text { mación obtenida }\end{array}$} & \multirow{5}{*}{\begin{tabular}{|l|} 
Desarrollar proce- \\
sos experimentales \\
(CI)
\end{tabular}} & \\
\hline & $\begin{array}{l}\text { Confección de } \\
\text { tablas }\end{array}$ & & & & \\
\hline & $\begin{array}{l}\text { Preparación de } \\
\text { disoluciones }\end{array}$ & & & & \\
\hline & $\begin{array}{l}\text { Realización de } \\
\text { observaciones con } \\
\text { el microscopio }\end{array}$ & & & & \\
\hline & $\begin{array}{l}\text { Descripción de } \\
\text { observaciones }\end{array}$ & & & & \\
\hline & $\begin{array}{l}\text { Interpretación de } \\
\text { los datos de una } \\
\text { observación }\end{array}$ & & $\begin{array}{l}\text { Procesar la infor- } \\
\text { mación obtenida }\end{array}$ & & \\
\hline & & & & $\begin{array}{l}\text { Manipular instru- } \\
\text { mentos de medida } \\
\text { (CI) }\end{array}$ & \\
\hline & & & & $\begin{array}{l}\text { Considerar nor- } \\
\text { mas de seguridad } \\
\text { en el laboratorio } \\
\text { (CI) }\end{array}$ & \\
\hline
\end{tabular}




\begin{tabular}{|c|c|c|c|c|c|}
\hline $\begin{array}{l}\text { Categorias del } \\
\text { instrumento de } \\
\text { evaluación NPTAI } \\
\text { (Ferrés et al.,2015) }\end{array}$ & $\begin{array}{l}\text { Categorias del } \\
\text { instrumento de } \\
\text { evaluación PTAI } \\
\text { (Tamir et al., } \\
\text { 1982) }\end{array}$ & $\begin{array}{l}\text { Diagrama de } \\
\text { investigación } \\
\text { (Gil, 1993) }\end{array}$ & $\begin{array}{l}\text { Competencias de la } \\
\text { dimensión metodo- } \\
\text { logica } \\
\text { (Pedrinaci et al., } \\
\text { 2012; Cañal, } \\
\text { 2012) }\end{array}$ & $\begin{array}{l}\text { Competencias bá- } \\
\text { sicas (CB) e investi- } \\
\text { gativas (CI) } \\
\text { (Garcia y Ladino, } \\
\text { 2008) }\end{array}$ & $\begin{array}{l}\text { Dimensiones de } \\
\text { la competencia } \\
\text { cientifica en la } \\
\text { investigación } \\
\text { (Franco-Mariscal) }\end{array}$ \\
\hline \multirow{8}{*}{$\begin{array}{l}\text { Análisis de datos } \\
\text { y obtención de } \\
\text { conclusiones } \\
\text { argumentadas }\end{array}$} & $\begin{array}{l}\text { Extracción de } \\
\text { conclusiones }\end{array}$ & & $\begin{array}{l}\text { Formular conclu- } \\
\text { siones fundamen- } \\
\text { tadas }\end{array}$ & & \multirow{6}{*}{$\begin{array}{l}\text { Análisis de datos } \\
\text { y emisión de } \\
\text { conclusiones }\end{array}$} \\
\hline & $\begin{array}{l}\text { Explicación de los } \\
\text { resultados de una } \\
\text { investigación }\end{array}$ & $\begin{array}{l}\text { Interpretación de } \\
\text { los resultados }\end{array}$ & & \multirow[t]{3}{*}{$\begin{array}{l}\text { Fomentar el } \\
\text { razonamiento } \\
\text { complejo (CI) }\end{array}$} & \\
\hline & $\begin{array}{l}\text { Análisis crítico de } \\
\text { los resultados }\end{array}$ & & & & \\
\hline & $\begin{array}{l}\text { Comprensión e } \\
\text { interpretación } \\
\text { de datos de un } \\
\text { gráfico }\end{array}$ & & & & \\
\hline & $\begin{array}{l}\text { Aplicación de } \\
\text { conocimientos }\end{array}$ & & & & \\
\hline & $\begin{array}{l}\text { Propuestas de } \\
\text { ideas para conti- } \\
\text { nuar la investiga- } \\
\text { ción }\end{array}$ & & & & \\
\hline & & $\begin{array}{l}\text { Comunicación } \\
\text { de los resultados } \\
\text { (intercambios con } \\
\text { otros equipos) }\end{array}$ & & $\begin{array}{l}\text { Emplear el lengua- } \\
\text { je científico para } \\
\text { comunicar las } \\
\text { ideas relacionadas } \\
\text { con la investiga- } \\
\text { ción (CB) }\end{array}$ & $\begin{array}{l}\text { Comunicación de } \\
\text { resultados de la } \\
\text { investigación }\end{array}$ \\
\hline & & & & $\begin{array}{l}\text { Trabajar en grupo } \\
\text { (CB) }\end{array}$ & \multirow{2}{*}{$\begin{array}{l}\text { Actitud/Reflexión } \\
\text { crítica y trabajo en } \\
\text { equipo }\end{array}$} \\
\hline Metarreflexión & & $\begin{array}{l}\text { Replanteamiento } \\
\text { de la investigación }\end{array}$ & & & \\
\hline
\end{tabular}

\section{UNA PROPUESTA ALTERNATIVA PARA DESARROLLAR COMPETENCIAS CIENTÍFICAS EN LA ENSEÑANZA-APRENDIZAJE POR INVESTIGACIÓN}

La propuesta que se realiza en este artículo parte de la consideración de la competencia científica como un conjunto de capacidades en relación con el conocimiento científico (Cañal, 2012) que el estudiante debe ser capaz de desarrollar, en este caso en el transcurso de una investigación escolar. Como sabemos, la competencia científica implica el dominio de conocimientos teóricos, conocimientos prácticos y actitudes, todos ellos presentes en la realización de un trabajo de investigación en la escuela. Asimismo, está de acuerdo con la definición de competencia de García y Ladino (2008), acorde con los objetivos de un trabajo de investigación en un modelo de enseñanza-aprendizaje por investigación, como "la posibilidad que debemos tener y manifestar para plantearnos problemas interesantes y para poder resolverlos a partir de entramados de conocimientos y de actitudes que se concretan en prácticas o esquemas de acción coherentes de dichos conocimientos, comunicando los resultados coherentemente a la comunidad científica», siempre refiriéndose al contexto escolar.

Seguidamente se realiza una propuesta para desarrollar competencias científicas en una enseñanzaaprendizaje por investigación, tomando como referencia los trabajos analizados en la tabla 1 y la ex- 
periencia del autor de este artículo en la coordinación de trabajos de investigación con estudiantes de secundaria.

De las distintas propuestas mostradas en la tabla 1, se puede inferir (ver última columna) que la competencia científica en una enseñanza-aprendizaje por investigación tiene siete dimensiones: planteamiento de la investigación; manejo de la información; planificación y diseño de la investigación; recogida y procesamiento de datos; análisis de datos y emisión de conclusiones; comunicación de resultados; y actitud o reflexión crítica y trabajo en equipo. Algunas de ellas son comunes a todos los estudios (recogida y procesamiento de datos, o análisis de datos y emisión de conclusiones), mientras que otras (comunicación de los resultados de la investigación o actitud-reflexión critica y trabajo en equipo), a pesar de que ciertos autores las consideren básicas, solo se incluyen en algunos de los trabajos.

A continuación, nos detendremos en caracterizar las capacidades de cuyo desarrollo progresivo depende el avance de la competencia científica, presentándolas en función de las dimensiones citadas:

Dimensión 1. Planteamiento de la investigación

La primera dimensión incluye tres capacidades: la capacidad del alumno para identificar e interesarse por problemas cientificos, la capacidad para definir los objetivos de una investigación y la capacidad para formular las hipótesis de la investigación. Como aportación, destaca incluir la capacidad para definir los objetivos de la investigación no recogidos en ninguno de los estudios anteriores como tales, y cuya explicitación por parte del alumno se considera fundamental en el planteamiento de la investigación.

Dimensión 2. Manejo de la información

Esta dimensión se corresponde con la capacidad del alumno para buscar información en diferentes fuentes y valorarla de forma critica y objetiva. A pesar de que esta competencia solo se recoge en el diagrama de Gil (1993), orientado hacia la toma de decisiones para definir una situación problemática, y en la dimensión actitudinal de Pedrinaci y Cañal (Pedrinaci et al., 2012; Cañal, 2012), creemos conveniente incluirla por dos razones. Por una parte, porque la búsqueda de la información y su valoración crítica es el eje sobre el que se sustenta el marco teórico y los antecedentes de una investigación que el estudiante debe dominar, y por otra parte, por la importancia cada vez mayor de Internet como fuente de información, que ya se sitúa, por encima de la televisión, como primera fuente de información científica de los ciudadanos (FECYT, 2012). La situación en la que actualmente se encuentra el estudiante cuando quiere informarse sobre un tema en Internet es la cantidad inabarcable de información disponible de diversa índole y calidad. Los ciudadanos deben disponer de conocimientos y capacidades para saber localizar, analizar y valorar estas informaciones. La capacidad de gestionar la información y el espíritu crítico ante ella son aspectos fundamentales de la competencia científica deseable para la ciudadanía (Blanco-López, España-Ramos, González-García y Franco-Mariscal, 2015), lo que refuerza la inclusión de esta capacidad en una enseñanza por investigación.

Dimensión 3. Planificación y diseño de la investigación

La tercera dimensión engloba tres capacidades relacionadas con la planificación y el diseño de la investigación: la capacidad para identificar las distintas variables de la investigación, la capacidad para diseñar una metodología de investigación y la capacidad para diseñar experiencias. Hemos creído conveniente incluir en esta dimensión, y no de manera independiente como hacen algunas de las propuestas analizadas, la capacidad para identificar variables por considerarla como el primer paso en la planificación de una investigación. Asimismo, se ha considerado relevante incluir una segunda capacidad en torno al diseño de la metodología de investigación, no denominada en estos términos en ninguno de los estudios previos, aunque se encuentre implícita en algunos de los ítems. Su importancia radica en que el alumno debe tener claro en cada momen- 
to qué pretende hacer y hacia dónde quiere dirigir su investigación, siempre previa consulta a su profesor. Asimismo se trata de una metodología sujeta a revisión a lo largo del proceso y que debe incluir la capacidad para diseñar experiencias, que por su importancia se propone como tercera capacidad de esta dimensión.

Dimensión 4. Recogida y procesamiento de datos

Esta dimensión, común a todos los trabajos analizados, incluye las capacidades relacionadas con los procesos más habituales en la recogida de datos de una investigación: la observación, el uso de instrumentos y el tratamiento de datos. En este sentido, el alumno debe desarrollar la capacidad para observar sistemáticamente, la capacidad para seleccionar y emplear el instrumento de medida más adecuado en cada caso, y la capacidad para procesar los resultados en distintos formatos (tablas, gráficas, etcétera).

Dimensión 5. Análisis de datos y emisión de conclusiones

La quinta dimensión incluye la capacidad para interpretar los resultados y la capacidad para formular conclusiones de la investigación que, por su relevancia dentro de una investigación, estaban presentes en todos los estudios seleccionados.

Dimensión 6. Comunicación de los resultados de la investigación

Esta dimensión, recogida solo en dos de los trabajos, incluye la capacidad para dar a conocer los resultados de la investigación. Es este un aspecto clave de una investigación, ya que si se hacen nuevos descubrimientos y no se dan a conocer al resto de la comunidad científica, la investigación no tendrá repercusión. Su traslado al contexto escolar consiste en que el estudiante sea capaz de realizar una difusión de su trabajo en diferentes ámbitos como exposiciones orales a sus compañeros, participación en ferias o certámenes de jóvenes investigadores, artículos, etcétera.

Dimensión 7. Actitud-reflexión crítica y trabajo en equipo

Por último, se incluye una dimensión en torno a la actitud y el trabajo en equipo que engloba la capacidad para reflexionar de forma critica sobre los resultados de la investigación y la capacidad para trabajar en equipo, respetar y valorar las ideas de los compañeros y tomar decisiones. Esta aportación se tiene en cuenta parcialmente en otros estudios -la dimensión actitudinal de Pedrinaci y Cañal (Pedrinaci et al., 2012; Cañal, 2012), el replanteamiento de la investigación (Gil, 1993), la metarreflexión de Ferrés et al. (2015) o el trabajo en equipo de García y Ladino (2008)-, pero no en conjunto como aquí se plantea.

La importancia de estas capacidades en un trabajo de investigación está reforzada con otros estudios que consideran estos aspectos clave como competencias necesarias para la ciudadanía. Así, la actitud crítica se entiende como una capacidad crítica en la que la persona es capaz de comprender y responder críticamente a temas científicos (Millar y Osborne, 1998), o como una habilidad en la que el alumno es capaz de aplicar algunas destrezas críticas como la observación o la argumentación para establecer sus conclusiones (Proyecto 2061, Asociación Americana para el Avance de la Ciencia, AAAS, 1989), como vemos en estrecha relación con una investigación. Respecto a la capacidad de trabajo en equipo, la National Council for Social Studies (NCSS, 1990) y Hurd (1998) indican su relación con la solución de problemas, mientras que el Proyecto DeSeCo (OCDE, 2002) menciona la cooperación y el trabajo en equipo como competencias que formarían parte de la interacción en grupos heterogéneos. En este sentido, los resultados de un reciente estudio Delphi para conocer cuáles son los aspectos clave de la competencia científica deseable para la ciudadanía (Blanco-López, España-Ramos, González-García y Franco-Mariscal, 2015), realizado con un amplio número de expertos españoles, indican con un alto grado de consenso estos dos aspectos que proponemos (actitud/espiritu crítico y trabajo en 
equipo), lo que refuerza aún más su integración como componentes de la competencia científica en una enseñanza-aprendizaje por investigación.

Finalmente, la tabla 2 recoge las distintas capacidades de cada una de las dimensiones de la competencia científica que un estudiante de secundaria debería ser capaz de desarrollar, en mayor o menor grado, en un trabajo de investigación según la propuesta presentada.

Tabla 2.

Distintas capacidades de cada dimensión de la competencia científica según la propuesta de Franco-Mariscal en una enseñanza-aprendizaje por investigación

\begin{tabular}{|c|c|}
\hline Dimensión & Capacidades \\
\hline 1. Planteamiento de la investigación & $\begin{array}{l}\text { Capacidad para identificar e interesarse por problemas científicos } \\
\text { Capacidad para definir los objetivos de una investigación } \\
\text { Capacidad para formular las hipótesis de una investigación }\end{array}$ \\
\hline 2. Manejo de la información & $\begin{array}{l}\text { Capacidad para buscar información en diferentes fuentes y valorarla de forma } \\
\text { crítica y objetiva }\end{array}$ \\
\hline $\begin{array}{l}\text { 3. Planificación y diseño de la investi- } \\
\text { gación }\end{array}$ & $\begin{array}{l}\text { Capacidad para identificar variables } \\
\text { Capacidad para diseñar una metodología de investigación } \\
\text { Capacidad para diseñar experiencias }\end{array}$ \\
\hline 4. Recogida y procesamiento de datos & $\begin{array}{l}\text { Capacidad para observar sistemáticamente } \\
\text { Capacidad para seleccionar y emplear el instrumento de medida más adecuado } \\
\text { Capacidad para procesar los resultados en distintos formatos (tablas, gráficas, etc.) }\end{array}$ \\
\hline $\begin{array}{l}\text { 5. Análisis de datos y emisión de con- } \\
\text { clusiones }\end{array}$ & $\begin{array}{l}\text { Capacidad para interpretar los resultados } \\
\text { Capacidad para formular conclusiones }\end{array}$ \\
\hline $\begin{array}{l}\text { 6. Comunicación de los resultados de } \\
\text { la investigación }\end{array}$ & Capacidad para dar a conocer los resultados \\
\hline $\begin{array}{l}\text { 7. Actitud-Reflexión crítica y trabajo } \\
\text { en equipo }\end{array}$ & $\begin{array}{l}\text { Capacidad para reflexionar de forma crítica sobre los resultados de la investigación } \\
\text { Capacidad para trabajar en equipo, respetar y valorar las ideas de los compañeros } \\
\text { y tomar decisiones }\end{array}$ \\
\hline
\end{tabular}

\section{UN ESTUDIO DE CASO SOBRE CORROSIÓN DE METALES}

Con idea de ejemplificar en un trabajo de investigación concreto las capacidades expuestas, se describe a continuación un estudio de caso sobre corrosión de metales realizado con estudiantes de secundaria obligatoria.

Este estudio de caso se llevó a cabo con un grupo de 10 estudiantes de 3..$^{\circ}$ e ESO (15 años) en el IES Juan Ramón Jiménez (Málaga) dentro del Programa Andalucía Profundiza (Junta de Andalucía, 2010), que fomenta la investigación entre el alumnado. Estos estudiantes presentaban interés por la Ciencia, tenían un rendimiento académico medio-alto y participaron en el programa de forma voluntaria en horario extraescolar durante cuatro meses, desde febrero hasta mayo del 2014, en sesiones de trabajo de tres horas de duración. Estos alumnos, trabajando en grupo y coordinados por el autor de este artículo, realizaron la investigación titulada Estudio del comportamiento corrosivo de aleaciones metálicas en una disolución de cloruro de sodio, que ha recibido tres premios educativos a nivel nacional en $2014 .{ }^{1}$

1. Esta investigación escolar ha recibido dos premios en el XXVII Certamen de Jóvenes Investigadores 2014 (Premio Especial de la Universidad Politécnica de Madrid al mejor trabajo de investigación en las áreas de Ingeniería y Tecnología, y Premio Representación de Espańa en el XXVII Certamen Europeo de Jóvenes Investigadores) y el Primer Premio de Ciencias en el nivel $2\left(1 .^{\circ}, 2 .^{\circ}\right.$ y $3 .^{\circ}$ de ESO) en los XX Premios San Viator de Investigación en Ciencias y Humanidades 2014. 
A continuación se presenta la metodología didáctica empleada por el profesor y las actividades realizadas por los estudiantes.

\section{Metodología didáctica}

La metodología utilizada por el profesor en el transcurso de la investigación se basó en el enfoque constructivista del aprendizaje (Driver y Oldham, 1986) y en las estrategias propias de la investigación como forma de aprendizaje (Gil et al., 1991), que se detalla seguidamente.

En primer lugar, para proporcionar una concepción preliminar de la tarea, generar el interés en los estudiantes, y encontrar un problema concreto para resolver se planteó la corrosión como tema de trabajo. A continuación, los alumnos trabajaron en grupo para estudiar de una forma cualitativa la situación problemática planteada. Con la ayuda de fotografías realizadas por ellos mismos en entornos cotidianos y de la bibliografía adecuada junto con unos criterios para valorarla (que se expondrán más adelante), los estudiantes comenzaron a delimitar el problema y a explicitar sus ideas.

El problema se abordó siguiendo una orientación científica con la definición de unos objetivos, la emisión de hipótesis, la explicitación de las ideas previas, la elaboración de posibles estrategias de resolución y análisis, y con la comparación de los resultados obtenidos por los distintos alumnos. En esta etapa surgió el conflicto cognitivo entre concepciones diferentes, lo que hizo posible emitir nuevas hipótesis o replantear el problema. Finalmente, se pretende que el estudiante maneje los nuevos conocimientos y que los aplique a nuevas situaciones cotidianas para profundizar en ellos y afianzarlos.

\section{Actividades desarrolladas por los estudiantes}

A partir de las dimensiones de la competencia científica expuestas, se indica cómo los alumnos desarrollaron las distintas capacidades en el transcurso de la investigación.

Dimensión 1. Planteamiento de la investigación

\section{Capacidad para identificar e interesarse por problemas cientificos}

La elección del tema de la investigación o del problema en torno al cual girará esta es un momento clave para que el alumnado desarrolle la capacidad para identificar e interesarse por un problema científico. En este caso, los alumnos optaron por intentar dar una respuesta a una serie de observaciones que habían realizado en las calles de su barrio. Concretamente, la presencia de mobiliario urbano de acero (señales de tráfico, rejas, alcantarillas, etcétera) totalmente oxidado, frente a otro mobiliario de aluminio, como ventanas o embellecedores de neumáticos de los vehículos, que apenas había sufrido este deterioro (figura 1). Observaciones como estas ponen de manifiesto el interés de los alumnos por intentar resolver un problema que afecta al medioambiente y produce pérdidas económicas en su ciudad, así como su capacidad para identificar este problema científico.

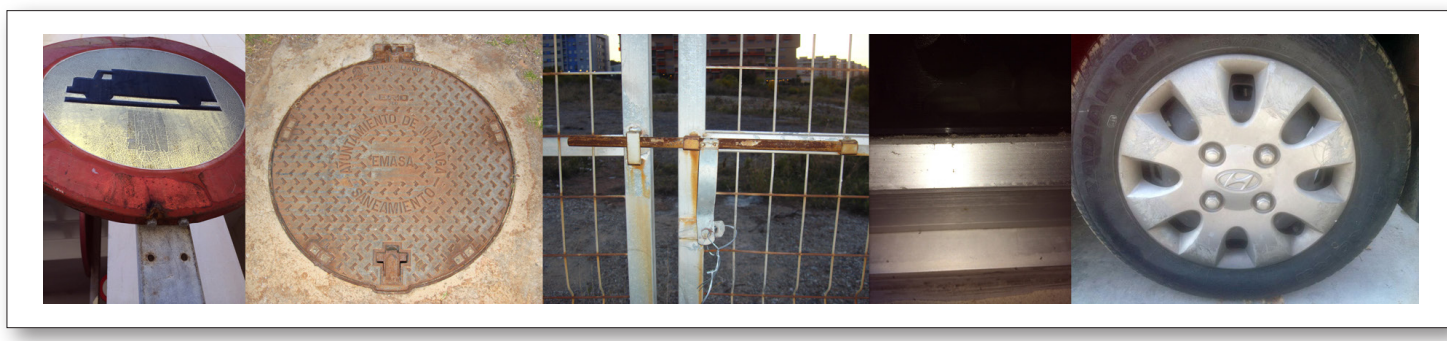

Fig. 1. Mobiliario urbano de acero y aluminio deteriorados por la corrosión. 


\section{Capacidad para definir los objetivos de una investigación}

Concretado el problema para investigar, el siguiente paso fue definir unos objetivos. En este momento, el profesor les indicó que sería interesante contactar con investigadores de la universidad que trabajasen en este tema con idea de obtener más información sobre el problema de la corrosión. Los investigadores de la Escuela de Ingeniería de la Universidad de Málaga les indicaron que tenía interés estudiar aceros y aluminios, al ser los materiales más comunes en construcción, y que debían elegir un medio para realizar el estudio. Esto les ayudó a definir el objetivo principal de la investigación como «estudiar de forma cualitativa y cuantitativa la corrosión de un acero convencional y una aleación de aluminio por inmersión en medio salino durante tres meses consecutivos a temperatura ambiente».

Los alumnos plantearon también otros objetivos específicos como conocer la fundamentación teórica del problema, investigar el efecto del cloruro de sodio y de la temperatura en la corrosión por comparación con resultados de inmersión en un blanco, optimizar un método de decapado para eliminar el óxido adherido a la superficie del metal y determinar la velocidad de corrosión.

La capacidad del alumnado para definir adecuadamente el objetivo principal de la investigación, así como otros objetivos secundarios, es muy importante ya que estos objetivos son los que guiarán posteriormente toda la investigación.

\section{Capacidad para formular las hipótesis de una investigación}

El siguiente paso fue formular varias hipótesis en torno a lo que creían que iba a suceder en la investigación. El desarrollo de esta competencia se puso de manifiesto ofreciendo hipótesis relacionadas con sus propias observaciones de la vida diaria como «los aceros se corroen más rápidamente que las aleaciones de aluminio» o "algunas disoluciones de uso cotidiano como el vinagre o la Coca Cola pueden eliminar el óxido como los decapantes propuestos en la bibliografía». Otras hipótesis se referían al efecto de ciertos factores en la velocidad de corrosión, como «la presencia de cloruros en el medio y la temperatura aceleran la corrosión».

Dimensión 2. Manejo de la información

\section{Capacidad para buscar información en diferentes fuentes y valorarla de forma critica y objetiva}

Antes de poder diseñar la parte experimental de la investigación, es imprescindible la búsqueda de información en diferentes fuentes para conocer el marco teórico del problema, así como sus antecedentes. Esta dimensión se considera muy importante, ya que pretende desarrollar en el estudiante la capacidad de valorar de forma crítica y objetiva la información que ha encontrado en Internet, y a partir de la cual debe construir el fundamento teórico de la investigación. Para ello, el profesor dio al alumnado una serie de criterios que facilitaron la valoración de la calidad y fiabilidad de la información en la web. Entre ellos, la identificación del autor de la web, valorándose más los contenidos realizados por responsables colectivos - centros de investigación o universidades- que por un individuo, y dentro de estos últimos los de mayor cualificación profesional; la inteligibilidad del mensaje, valorándose positivamente redacciones adecuadas tanto a nivel ortográfico como gramatical, y la distinción entre información y opinión; la independencia del mensaje, en el que se valora especialmente la separación de la información de posibles mensajes publicitarios contenidos en la web, entre otros (Fornás, 2003; Franco, España y Blanco, 2014).

Esto permitió que los alumnos desarrollasen adecuadamente esta capacidad y que construyeran un marco teórico sólido que incluyó información sobre el hierro y el aluminio y sus aleaciones, así como 
sobre el proceso de corrosión que ataca a las aleaciones en su superficie de forma química o electroquímica por diferentes medios, como la atmósfera o el agua, produciendo pérdidas de material que en ocasiones puede llegar hasta su destrucción total. Asimismo, incluyeron información sobre los tipos de corrosión (uniforme o localizada), sus mecanismos, la operación de decapado y los métodos para calcular la velocidad de corrosión.

La dimensión de la competencia científica que aquí se aborda permitió también al alumnado elaborar los antecedentes de la investigación y conocer los trabajos de algunos grupos de investigación que estudian este tema.

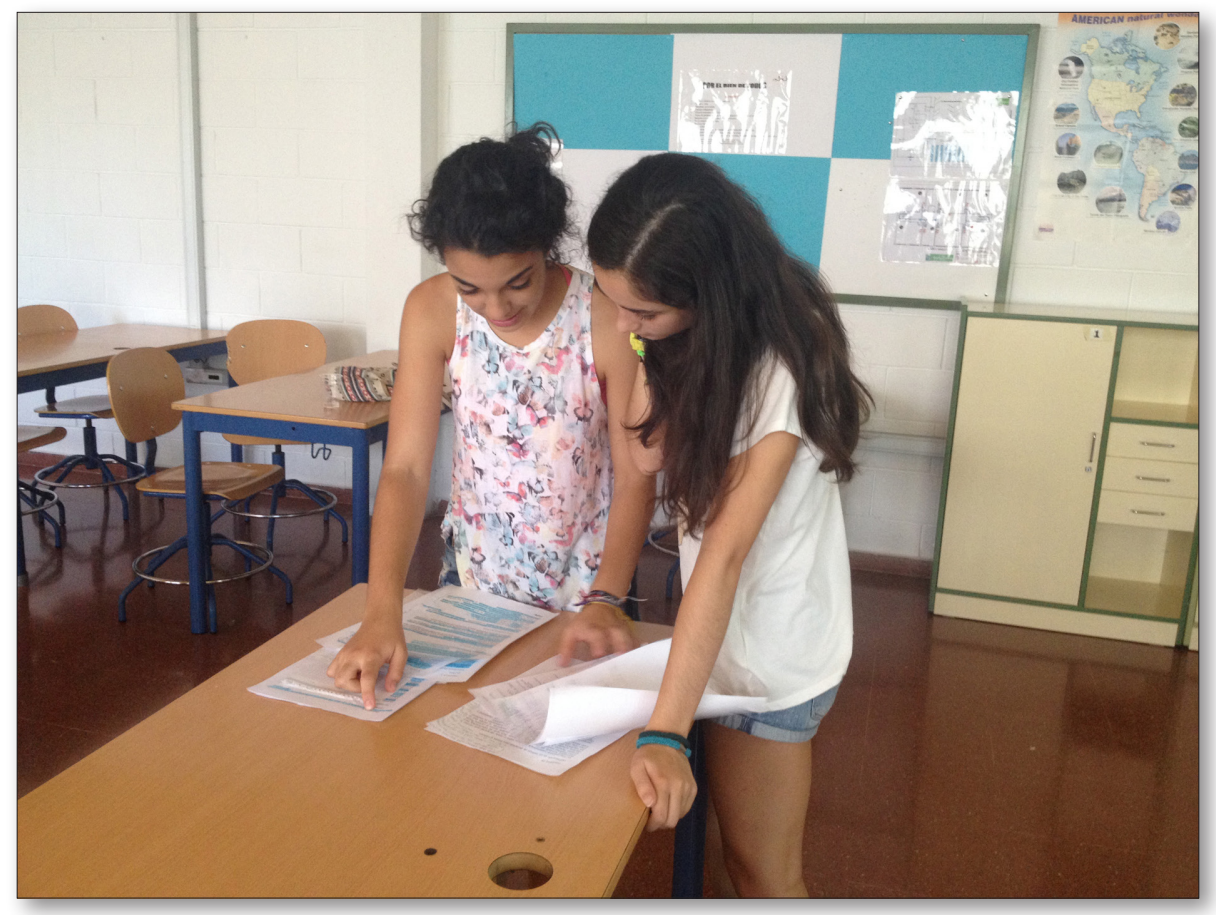

Fig. 2. Dos estudiantes valoran la calidad de la información buscada.

Dimensión 3. Planificación y diseño de la investigación

\section{Capacidad para identificar variables}

Como se indicó anteriormente, la identificación de variables debe ser la primera etapa en la planificación de una investigación. De esta forma, los estudiantes constataron, con ayuda de su profesor, que en el problema para investigar había un conjunto amplio de variables implicadas: la naturaleza de la aleación -solo de aceros y aluminios podemos encontrar un elevado número de aleaciones de diferente composición química-, el medio en el que tiene lugar la corrosión (la atmósfera o una disolución) o la temperatura, por lo que había que realizar una primera toma de decisiones y fijar variables para acotar la investigación. Se optó por trabajar con dos aleaciones -el acero al carbono F111 y el aluminio 2030-, emplear como medio agresivo una disolución de cloruro de sodio al 3,5\% en masa, que simula la concentración del agua de mar, y estudiar dos temperaturas, ambiente y $100{ }^{\circ} \mathrm{C}$. 


\section{Capacidad para diseñar una metodología de investigación}

Identificadas las variables y seleccionadas las muestras, procede diseñar una metodología de trabajo. Esta capacidad se puso de manifiesto mediante una metodología que tuvo en cuenta un tratamiento previo a las muestras, el diseńo de distintas series de experimentos que permitieron la comparación de resultados, el diseńo de un protocolo de trabajo, y una selección adecuada de instrumentos y fórmulas para determinar tanto de manera cualitativa como cuantitativa la corrosión de las muestras estudiadas.

Para ello, los alumnos, con ayuda de su profesor, propusieron las siguientes etapas de la metodología de la investigación:

1. Tratamiento previo de limpieza (lijado, lavado y secado) y determinación de parámetros (masa, dimensiones, superficie, volumen y densidad) de las muestras iniciales (figura 3).

2. Diseño de experimentos (ver capacidad siguiente).

3. Diseño de un protocolo de trabajo en los experimentos (ver dimensión 4).

4. Determinación cualitativa y cuantitativa de la velocidad de corrosión. La corrosión se estudió de forma cualitativa a través de un estudio morfológico de la superficie de las muestras con microscopía óptica, y de forma cuantitativa a partir de la pérdida de masa por unidad de tiempo y de superficie, y a través de la disminución de espesor.

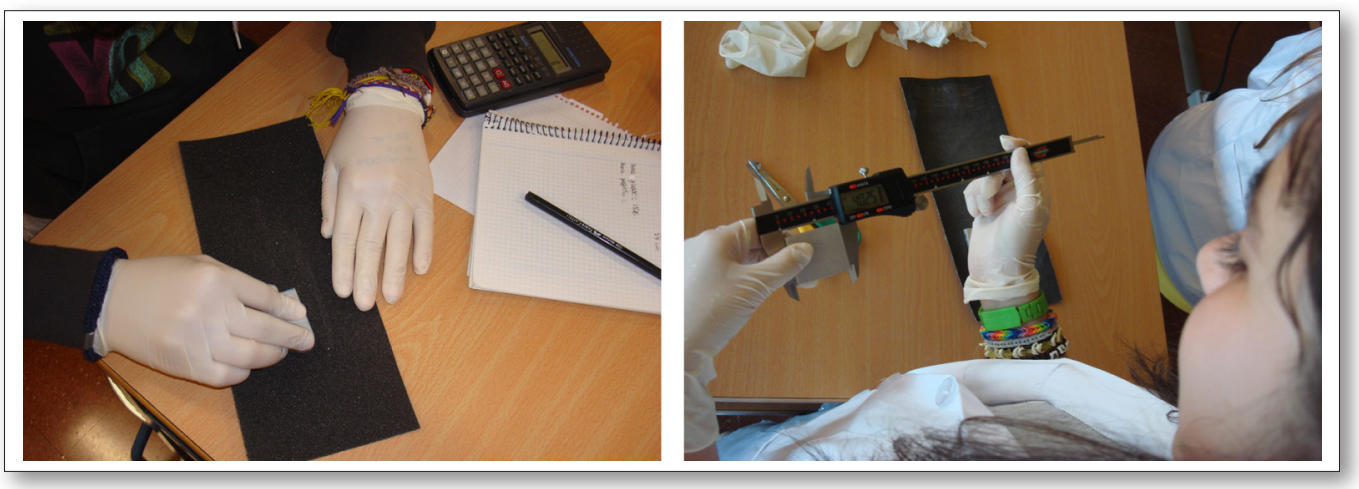

Fig. 3. Tratamiento previo de lijado de la aleación (imagen izquierda), y alumna determina las dimensiones de una muestra con un calibre digital (imagen derecha).

\section{Capacidad para diseñar experiencias}

Los estudiantes desarrollaron esta capacidad diseñando dos tipos de experimentos: inmersión y decapado. Los experimentos de inmersión permitieron estudiar los cambios que se producen en los aceros y las aleaciones de aluminio sumergidas en una disolución de cloruro de sodio durante 90 días consecutivos, a temperatura ambiente, o durante 90 minutos a $100^{\circ} \mathrm{C}$. Otra serie de experimentos estudió la inmersión en agua de baja mineralización utilizada como blanco para comprobar el efecto de los cloruros en el medio. Los alumnos estudiaron la evolución de estos cambios en muestras sometidas a inmersión durante 15, 30, 45, 60, 75 y 90 días.

Los experimentos de decapado permitieron encontrar el mejor método para eliminar el óxido adherido a la superficie de los metales tras la corrosión y poder determinar posteriormente la velocidad de corrosión de forma precisa. Para ello, se utilizaron disoluciones cotidianas como Coca Cola, vinagre o agua fuerte, de reconocido efecto decapante, y otras más específicas como ácido nítrico. Una vez decidido el mejor método, se realizó el decapado de todas las muestras. 
Algunas evidencias del desarrollo de esta capacidad fueron la adecuada selección de parámetros en el estudio (tiempos de inmersión o temperaturas) o de decapantes cotidianos, la inclusión en el diseño de un experimento blanco con agua de baja mineralización para conocer el efecto de los iones cloruro en la corrosión, o que algunos alumnos propusieran la realización de los experimentos por duplicado para obtener reproducibilidad.

\section{Dimensión 4. Recogida y procesamiento de datos}

\section{Capacidad para observar sistemáticamente}

Durante la recogida de datos es de suma importancia desarrollar la capacidad de observación sistemática, y para ello resulta conveniente haber diseñado previamente un protocolo de actuación. En nuestro caso, los estudiantes desarrollaron esta capacidad siguiendo un protocolo que permitía tanto la reproducibilidad en la toma de datos (etiquetar los vasos, utilizar el mismo volumen de disolución rellenando con agua de baja mineralización cuando se evaporase, y sumergir totalmente las muestras sin tocar las paredes del recipiente), como la obtención de información tanto del metal como de la disolución (hacer una descripción cualitativa de las muestras y de la disolución cada varios días, hacer un seguimiento fotográfico, determinar la masa cada 15 días o medir el $\mathrm{pH}$ ).

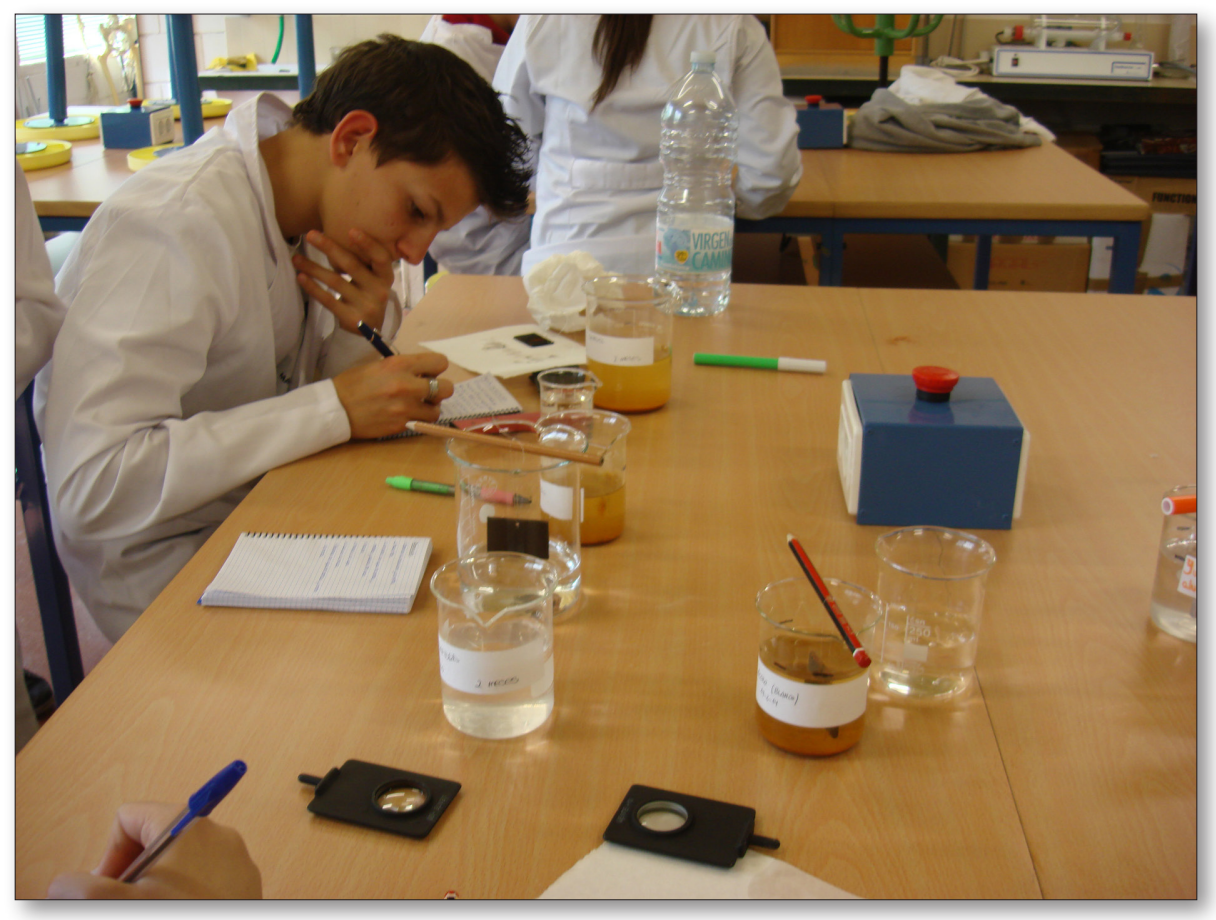

Fig. 4. Un alumno observa los cambios producidos en las distintas muestras.

Este protocolo permitió observar los cambios en las aleaciones, tras su inmersión en la disolución, más significativos al incrementar el tiempo y la temperatura y ante la presencia de cloruros. A modo de ejemplo, se ofrece la siguiente descripción realizada por uno de los alumnos en su cuaderno de laboratorio: «La muestra de acero que lleva 90 días en inmersión ha sufrido una pérdida de brillo considerable, tiene una capa de óxido de color negro sobre su superficie y ha vuelto a perder masa. No se observan picaduras. La disolución está cada vez más naranja y hay más precipitado en el fondo del vaso. El pH sigue siendo 7,3». La tabla 3 muestra con imágenes estos cambios en función del tratamiento. 
Tabla 3.

Observaciones realizadas en los experimentos de inmersión de aceros

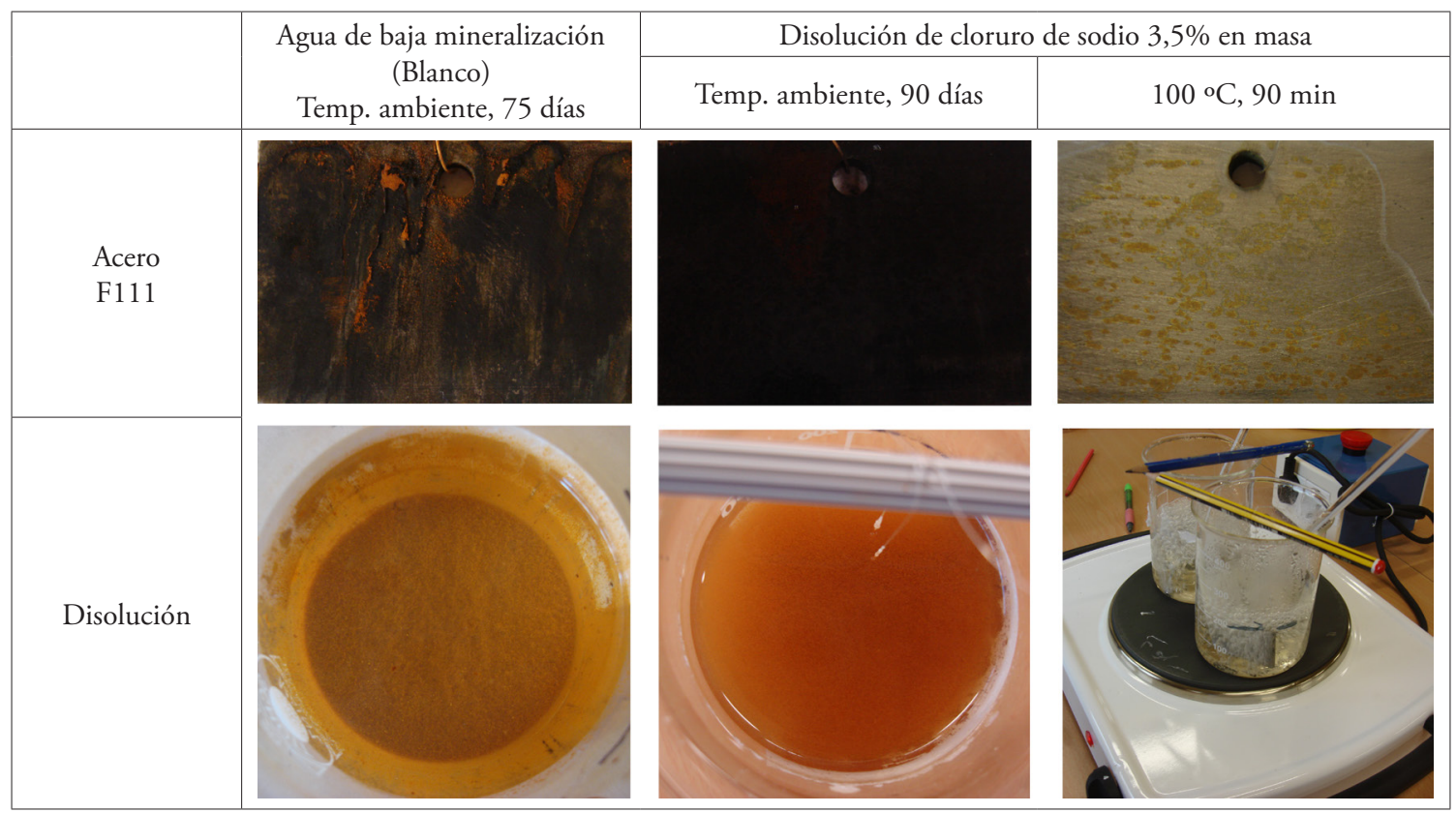

\section{Capacidad para seleccionar y emplear el instrumento de medida más adecuado}

Un ejemplo del desarrollo de esta capacidad por parte del alumnado fue la utilización de los siguientes instrumentos de gran precisión: un calibre digital en lugar de una regla para medir las dimensiones de los metales, una balanza digital $( \pm 0,01 \mathrm{~g})$ para determinar las pérdidas de masa de las aleaciones, o un microscopio óptico (160 aumentos) para estudiar la morfología de la superficie (figura 5).

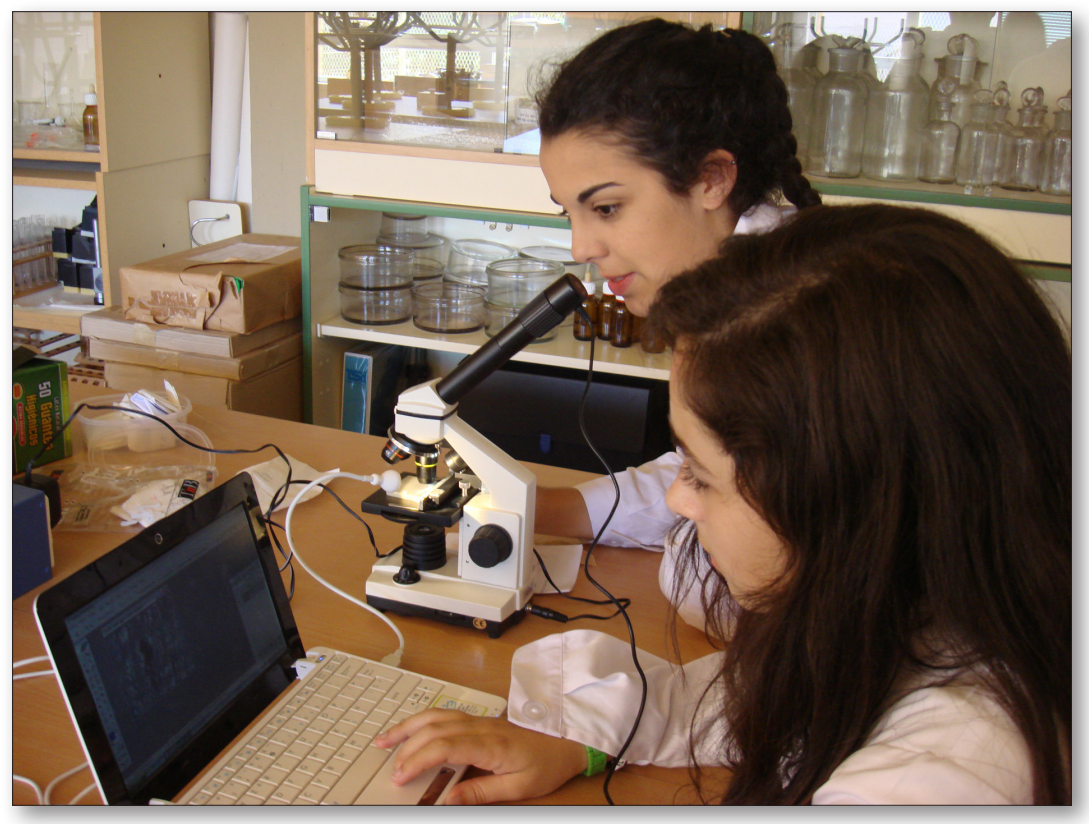

Fig. 5. Dos alumnas utilizan el microscopio óptico. 
Capacidad para procesar los resultados en forma de tablas, gráficas, etcétera

El elevado número de muestras implicadas en la investigación y la gran cantidad de datos asociados a cada una hizo obligatorio representar la información en diferentes tablas y gráficas que permitieran la comparación de los datos, fomentándose así la adquisición de esta capacidad. A modo de ejemplo, la figura 6 recoge la gráfica realizada por los estudiantes para conocer la evolución de la velocidad de corrosión del acero con el tiempo tras su inmersión a temperatura ambiente en una disolución de sal común y posterior decapado con vinagre. A partir de estos datos, realizaron un ajuste por mínimos cuadrados comprobando que existía una relación lineal entre velocidad de corrosión y temperatura.

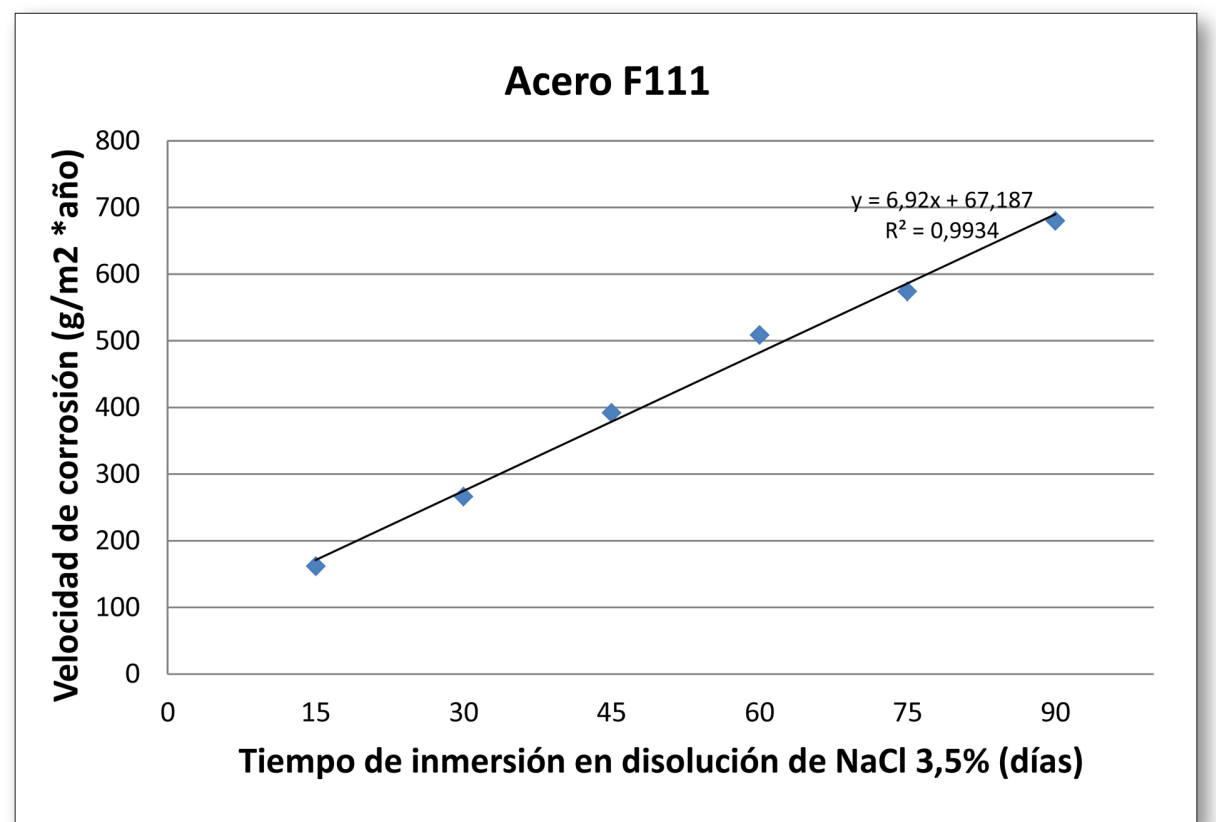

Fig. 6. Velocidad de corrosión del acero F111 con el tiempo tras inmersión en cloruro de sodio a temperatura ambiente y decapado con vinagre.

Dimensión 5. Análisis de datos y emisión de conclusiones

\section{Capacidad para interpretar los resultados}

La dificultad del alumnado para desarrollar esta capacidad se puso de manifiesto en la interpretación de los resultados de los experimentos con distintas disoluciones decapantes, especialmente en la toma de una decisión acerca de cuál era el mejor decapante para eliminar el óxido, ya que había muchos factores implicados. En esta decisión, los alumnos tuvieron que poner en tesitura varias variables como el tiempo necesario, la limpieza producida por el decapante, la pérdida de masa o el ataque superficial. En el caso del acero, consideraron el vinagre ( $6 \%$ acético) como un método recomendado ( 1 hora), la Coca Cola como un método apto (1 hora) y el agua fuerte $(12 \% \mathrm{HCl})$ y ácido nítrico $(15 \%)$ como métodos no recomendados. Los estudiantes se apoyaban en argumentos como estos: «El vinagre produce una limpieza importante y una pérdida de masa sin ataque a la superficie en relativamente poco tiempo», o «no recomiendo el agua fuerte o el ácido nítrico porque producen ataques importantes a la superficie de la muestra». 


\section{Capacidad para formular conclusiones}

El análisis adecuado de los datos permitió al alumnado extraer como conclusión de la investigación que «el acero F111 se corroe más rápidamente que la aleación de aluminio 2030 inmersa en cloruro de sodio a temperatura ambiente, siendo la velocidad de corrosión del acero tras 90 días de inmersión de $680,1 \mathrm{~g} / \mathrm{m}^{2 *}$ año, y su disminución de espesor de $0,09 \mathrm{~mm} /$ año, mientras que no se ha podido determinar experimentalmente la velocidad de corrosión del aluminio 2030». Asimismo, los estudiantes emitieron varias conclusiones específicas relativas a los distintos aspectos que se habían trabajado a lo largo de la investigación, como que «la temperatura acelera el proceso de corrosión de ambos metales» o «en el acero F111 la corrosión puede considerarse uniforme, y en el aluminio 2030 la corrosión es localizada por picaduras».

\section{Dimensión 6. Comunicación de resultados}

\section{Capacidad para comunicar o dar a conocer los resultados}

Al finalizar la investigación, los estudiantes tuvieron la oportunidad de dar a conocer los resultados obtenidos a varias audiencias mediante exposiciones orales ayudados con una presentación de diapositivas. Por un lado, al resto de la comunidad educativa del centro, y por otro, ante otros jóvenes investigadores en el Certamen Jóvenes Investigadores 2014 en el que tres de los alumnos fueron galardonados con dos premios. En ambos casos, tras la exposición tuvieron lugar interesantes debates entre los jóvenes.

Comunicar ideas es importante porque lleva implícito una síntesis de todo el trabajo. De este modo, el alumno que sea capaz de comunicar con éxito habrá trabajado otras competencias clave, además de la científica. La exposición permite de este modo desarrollar la competencia lingüística al expresarse oralmente y sintetizar ideas en las diapositivas, la competencia digital al utilizar una presentación, y en definitiva, también habrá desarrollado el hecho de aprender a aprender o la autonomía e iniciativa personal. Asimismo, el debate le permite desarrollar la competencia social y ciudadana.

\section{Dimensión 7. Actitud-reflexión crítica y trabajo en equipo}

\section{Capacidad para reflexionar de forma critica sobre los resultados de la investigación}

La investigación realizada ha dado también la oportunidad de desarrollar una actitud o reflexión crítica. Por un lado, comprendiendo en profundidad el tema estudiado a la vez que se crea una toma de conciencia hacia un problema medioambiental, y por otro lado, desarrollando destrezas críticas para poder ofrecer argumentos que conduzcan a unas conclusiones sólidas.

\section{Capacidad para trabajar en equipo, respetar y valorar las ideas de los compañeros y tomar decisiones}

Por último, el trabajo en equipo ha sido un aspecto fundamental en esta investigación, especialmente por el elevado número de muestras y datos para recopilar, y cuyo seguimiento hubiera sido muy complicado de hacer solo por unas pocas personas. Esto contribuyó a que el reparto de tareas fuera equilibrado y a la necesidad del intercambio de información periódica entre todos los alumnos. Estas puestas en común permitieron comparar cualitativamente las muestras y ofrecer distintos puntos de vista a los resultados que se estaban obteniendo, lo que contribuyó a desarrollar la capacidad de respeto y valoración de otras ideas para la toma de decisiones. 


\section{CONSIDERACIONES FINALES}

Este artículo ha planteado un enfoque alternativo para el desarrollo de la competencia científica en una enseñanza-aprendizaje de las Ciencias por investigación en la educación secundaria, partiendo de las aportaciones de otros trabajos relevantes y de la experiencia del autor en trabajos de investigación con alumnos.

El enfoque considera que la competencia científica tiene siete dimensiones: planteamiento de la investigación; manejo de la información; planificación y diseño de la investigación; recogida y procesamiento de datos; análisis de datos y emisión de conclusiones; comunicación de resultados y actitudreflexión crítica y trabajo en equipo. Todos ellos, aspectos importantes que deben formar parte de la competencia científica.

La propuesta resalta algunos aspectos que a menudo no son suficientemente tenidos en cuenta en la enseñanza de las Ciencias, y que consideramos fundamentales dentro de una investigación escolar como el manejo de la información, la comunicación de resultados y la actitud-reflexión crítica y el trabajo en equipo. Si bien es cierto que algunos de los trabajos analizados los tienen en cuenta, solo lo hacen de una forma parcial. Asimismo, se ha intentado mostrar, a través de un estudio de caso, cómo los estudiantes de secundaria pueden desarrollar con éxito estas capacidades.

A pesar de la potencialidad de los trabajos de investigación en la escuela, no debemos olvidar las dificultades con las que se encuentra el alumnado para desarrollar y dominar todas estas dimensiones de la competencia científica. Entre ellas, se encuentra la metodología habitual de gran parte del profesorado centrada en el currículo tradicional y muy alejada de investigaciones verdaderas, o el curso en el que la mayoría de los alumnos empieza a desarrollar estas investigaciones, bachillerato o $4 .^{\circ}$ de ESO en el mejor de los casos. Por ello sería interesante que el alumno pudiera adquirir estas capacidades poco a poco desde los primeros cursos de secundaria. De acuerdo con Menoyo (2013), sería recomendable iniciar los trabajos de investigación con alumnos en $1 .^{\circ}$ de ESO consensuado entre los profesores de la etapa y desde las distintas materias.

Es importante resaltar que estas competencias científicas se pueden desarrollar independientemente de que el trabajo de investigación que se esté realizando sea o no de Ciencias, ya que se trata de unas capacidades ligadas a la metodología científica, y por tanto, aplicables desde cualquier área.

Finalmente, queremos indicar que, aunque el estudio se ha realizado con un pequeño grupo de estudiantes en horario extraescolar, creemos que es posible generalizar esta forma de trabajar. De este modo, tanto docentes como alumnos saldrían beneficiados si se dieran a conocer al profesorado más ejemplos de buenas prácticas en este tipo de enseñanza, así como si este tipo de tareas formasen parte de los programas de formación inicial y permanente del profesorado.

\section{REFERENCIAS BIBLIOGRÁFICAS}

AAAS, Asociación Americana para el Avance del Ciencia (1989). Science for All Americans. Disponible en línea: <http://www.project2061.org/publications/sfaa/online/sfaatoc.htm> (consulta: 30-0315).

Bell, R.; Maeng, J. y Peters, E. (2010). VMSC Scientific Inquiry and NOS Task Force Report. Virginia. Belmonte, M. (2011). Enseñar a investigar. Libro del profesorado. Bilbao: Mensajero.

Blanco-López, A.; España-Ramos, E.; González-García, F.J. y Franco-Mariscal, A. J. (2015). Key Aspects of Scientific Competence for Citizenship: A Delphi Study of the Expert Community in Spain. Journal of Research in Science Teaching, 52(2), pp. 164-198.

http://dx.doi.org/10.1002/tea.21188 
Burbules, N. y Linn, M. (1991). Science education and philosophy of science: congruence or contradiction? International Journal of Science Education, 13(3), pp. 227-241. http://dx.doi.org/10.1080/0950069910130302

CaAmaño, A. (2012). ¿Cómo introducir la indagación en el aula? Alambique. Didáctica de Las Ciencias Experimentales, 70, pp. 83-91.

Cañal, P. (2012). ¿Cómo evaluar la competencia científica? Investigación en la Escuela, 78, pp. 5-17.

Driver, R. y Oldham, V. (1986). A constructivist approach to curriculum development in science. Studies in Science Education, 13(1), pp. 105-122.

http://dx.doi.org/10.1080/03057268608559933

FECYT (2012). VI Encuesta de Percepción Social de la Ciencia 2012. Disponible en línea: <http://www. fecyt.es/fecyt/docs/tmp/363174605.pdf> (consulta: 30-03-15).

Fernández-López, L. (coord.) (2011). Los proyectos de investigación del alumnado y las competencias básicas y científicas. En Cuaderno de indagación en el aula y competencia científica. Madrid: Ministerio de Educación.

Ferrés, C.; Marbà, A. y Sanmartí, N. (2015). Trabajos de indagación de los alumnos: Instrumentos de evaluación e identificación de dificultades. Revista Eureka sobre Enseñanza y Divulgación de las Ciencias, 12(1), pp. 22-37.

Fornás, R. (2003). Criterios para evaluar la calidad y fiabilidad de los contenidos en Internet. Rev. Esp. Doc. Cient., 26(1), pp. 75-80.

Franco-Mariscal, A. J. (2015). Investigación con helados para caracterizar las propiedades de la materia. Alambique, Didáctica de las Ciencias Experimentales, 80, pp. 73-82. http://dx.doi.org/10.5565/rev/ensciencias.1346

Franco-Mariscal, A. J.; Blanco-López, A. y España-Ramos, E. (2014). El desarrollo de la competencia científica en una unidad didáctica sobre la salud bucodental. Diseño y análisis de tareas. Enseñanza de las Ciencias, 32(3), pp. 649-667.

Franco-Mariscal, A. J.; España-Ramos, E. y Blanco-López, A. (2014). Uso de Internet para analizar las relaciones entre drogas y salud bucodental. Una experiencia en 4. ${ }^{\circ}$ de ESO. En Soriano, E.; González, A. J. y Cala, V.C. (eds.): Retos actuales de educación y salud transcultural [1]. Almería (España): Universidad de Almería, pp. 54.1-54.8.

García, G. A. y Ladino, Y. (2008). Desarrollo de competencias científicas a través de una estrategia de enseñanza y aprendizaje por investigación. Studiositas, 3(3), pp. 7-16.

GiL, D. (1986). La metodología científica y la enseñanza de las ciencias. Unas relaciones controvertidas. Enseñanza de las Ciencias, 4(2), pp. 111-121.

GiL, D. (1993). Contribución de la historia y de la filosofía de las ciencias al desarrollo de un modelo de enseñanza/aprendizaje como investigación. Enseñanza de las Ciencias, 11(2), pp. 197-212.

Gil, D.; Carrascosa, J.; Furió, C. y Martínez-Torregrosa, J. (1991). La enseñanza de la ciencia en la educación secundaria. Barcelona: ICE, Universidad Autónoma de Barcelona.

Hurd, P. D. (1998). Scientific literacy: new minds for a changing world. Science Education, 82(3), pp. 407-416.

http://dx.doi.org/10.1002/(SICI)1098-237X(199806)82:3<407::AID-SCE6>3.3.CO;2-Q http://dx.doi.org/10.1002/(SICI)1098-237X(199806)82:3<407::AID-SCE6>3.0.CO;2-G

JimÉnez-AleiXANDRE, M. P. (2009). Competencia científica: poner en práctica los saberes de ciencias. Presentación del monográfico sobre competencia científica. Aula de Innovación Educativa, 186, p. 6.

Junta de Andalucía, Consejería de Educación (2010). Programa Andalucía Profundiza. Disponible en línea: <http://profundiza.es/> (consulta: 30-03-2015).

MEC, Ministerio de Educación y Ciencia (2006). Real Decreto 1513/2006, de 7 de diciembre, por el que se establecen las enseñanzas mínimas correspondientes a la Educación Primaria (BOE núm. 293, 8 de diciembre de 2006). 
MEC, Ministerio de Educación y Ciencia (2007). Real Decreto 1631/2006, de 26 de diciembre, por el que se establecen las enseñanzas mínimas correspondientes a la Educación Secundaria Obligatoria (BOE núm. 5, 5 de enero de 2007).

Menoyo, M. P. (2013). Anàlisi del procés de realització i tutorització dels treballs de recerca a secundària. Tesis Doctoral Universitat Autònoma de Barcelona.

Millar, R. y Osborne, J. (eds.) (1998). Beyond 2000: Science education for the future. London. King's College. School of Education.

http://dx.doi.org/10.1038/32514

http://dx.doi.org/10.1038/25290

NCSS, National Council for Social Studies (1990) (Eds. Health, P. et al.). Teaching about Science, Technology, and Society in Social Studies: Education for Citizenship in the 21st Century. Social Education, 54(4), April-May, pp. 189-193.

OCDE (2002). Definition and Selection of Competences (DeSeCo): theoretical and conceptual foundations. OCDE: París. Disponible en línea: <http://www.deseco.admin.ch/bfs/deseco/en/index/02.html> (consulta: 30-03-15).

OCDE (2006). PISA 2006. Marco de la evaluación, conocimientos y habilidades en ciencias, matemáticas y lectura. Madrid: Santillana.

OCDE (2009). PISA 2009. Assessment framework. Key competencies in reading, mathematics and science. Disponible en línea: <www.oecd.org/dataoecd/11/40/44455820.pdf> (consulta: 30-03-15).

Pedrinaci, E.; CaAmaño, A.; Cañal, P. y Pro, A. (2012). La evaluación de la competencia científica requiere nuevas formas de evaluar los aprendizajes. En Pedrinaci, E. (coord.). 11 ideas clave: El desarrollo de la competencia cientifica. Barcelona: Graó, cap. 11, pp. 241-267.

Pro, A. (2011). Conocimiento científico, ciencia escolar y enseñanza de las ciencias en la educación secundaria. En Caamaño, A. (coord.). Didáctica de la Física y la Quimica. Barcelona: Graó-Me, pp. 13-33.

Rocard, M. (2007): Science Education Now: A Renewed Pedagogy for the Future of Europe. Bruselas: Comisión Europea. [Resumen en castellano en Alambique, Didáctica de las Ciencias Experimentales, 55, pp. 104-120].

SAnMarTí, N. (2010). Competencias: ¿más burocracia o un constructo útil? II Congreso Internacional de Didácticas. La actividad del docente: Intervención, innovación, investigación. Girona, Febrero 2010, pp. 51-66.

Tamir, P. y García, M. (1992). Características de los ejercicios de prácticas de laboratorio incluidos en los libros de texto de ciencias utilizados en Cataluña. Enseñanza de las Ciencias, 10(1), pp. 3-12.

Tamir, P.; Nussinovitz, R. y Friedler, Y. (1982). The development and use of a Practical Test Assessment Inventory. Journal of Biological Education, 16, pp. 42-50. http://dx.doi.org/10.1080/00219266.1982.9654417

UE, Unión Europea (2006). Recommendation 2006/962/EC of the European Parliament and of the Council, of 18 December, on key competences for lifelong learning. Brussels. Disponible en línea: <http://eur-lex.europa.eu/legal-content/EN/TXT/PDF/?uri=CELEX:32006H0962\&from=EN> (consulta: 26-12-14).

VARGaS, F. (2002). Competencia en la formación y competencia en la gestión del talento humano. Convergencias y desafíos. CINTERFOR-OIT.

VIlches, A. y Gil, D. (2010). El programa PISA: un instrumento para la mejora del proceso de enseñanza-aprendizaje. Revista Iberoamericana de Educación, 53, pp. 121-154.

Wheatley, G. H. (1991). Constructivistperspectives on Science and Mathematics leaming. Science Education, 75(1), pp. 9-21.

http://dx.doi.org/10.1002/sce.3730750103 


\title{
Scientific competences in teaching and learning through research. A case study about the corrosion of metals in secondary education
}

\author{
Antonio Joaquín Franco-Mariscal \\ IES Juan Ramón Jiménez, Málaga, España. \\ Universidad de Málaga, Málaga, España. \\ antoniojoaquin.franco@uma.es
}

The current framework in education pays special attention to the development of key competences in high school students. Competences are defined by the European Union as a combination of knowledge, skills and attitudes appropriate to the context. Among these key competences is the scientific competence.

Various ways to understand and characterize the scientific competence have been proposed in the teaching of science, among which the approaches of PISA, Spanish curricula, and the recent contribution of Pedrinaci and Cañal have stood out. All these contributions believe desirable to develop some abilities in students related to research in the school context.

In this sense, inquiry is one of the most fruitful and debated research areas in science education. The teachlearning of science based on the model of learning research offers students a closer knowledge as scientists do in a school context and facilitates the development of scientific competences.

A review of the different proposals about scientific competences in school researches is analyzed in this paper and an alternative approach to the development of the scientific competence in teaching and learning through research in secondary education is presented from that analysis. According to our approach, the scientific competence has seven dimensions: (1) proposal of research, (2) information management, (3) planning and research design, (4) collection and processing of data, (5) data analysis and drawing conclusions, (6) communication of results, and (7) critical attitude/thinking and ability to work as a part of a team. Some aspects like information management, communication of results, and critical attitude/thinking and ability to work as a part of a team, which are often not sufficiently considered in the teaching of science, are emphasized in this proposal. Our approach includes the following competences: ability to identify and interest in scientific issues, ability to define research objectives, ability to formulate hypotheses (dimension 1), ability to search information from different sources and evaluate it critically and objectively (dimension 2), ability to identify variables, ability to design a research methodology, ability to design experiments (dimension 3), ability to systematically observe, ability to select and use the most appropriate measurement instrument, ability to process results in various formats (dimension 4), ability to interpret results, ability to formulate conclusions (dimension 5), ability to publish results (dimension 6), ability to reflect critically on the research results, and ability to work as a part of a team, respecting and valuing other ideas and taking decisions (dimension 7).

The approach is illustrated with examples in a case study with 15 year-old Spanish compulsory secondary students where pupils research about corrosion. This school research was made in Andalucia Profundiza program in 2014 and has obtained three awards at national level. Finally, some considerations about the importance of the approach and the need of a teacher training in school research works are made. 